\title{
Estratégias de operações de serviço no setor público: um exame a partir da produção científica brasileira no período de 2004-2014
}

O presente artigo tem como objetivo realizar uma revisão sistemática do campo de Gestão de Operações de Serviços (GOS) no setor público, no período de 2004 a 2014, a fim de verificar as características e evolução das publicações nacionais na área. Os resultados da pesquisa foram divididos em resultados descritivos e resultados temáticos. Os descritivos buscaram responder quatro questões adaptadas do modelo proposto por Machuca et al. (2007): (A) que variações, se houver, podem ter ocorrido no número de publicações em Operações de Serviço no setor público no decorrer dos anos? (B) quais são os principais temas de pesquisa? (C) que métodos são usados em pesquisas de GOS no setor público? (D) quais são os setores de atividade mais estudados? Os resultados temáticos se basearam na classificação dos artigos segundo seu tópico focal - conteúdo ou processo - conforme apresentado por Dangayach e Deshmukh (2001) e Boyer et al. (2005). Por meio de resultados descritivos, verificou-se uma distribuição de trabalhos voltados para o setor público acompanhando as publicações gerais de GOS, tendo como principal tema abordado o planejamento, a modelagem como método mais utilizado e o setor de saúde como o mais estudado. Quanto aos resultados temáticos percebeu-se uma concentração de artigos que focam aspectos de conteúdo com perceptível destaque para as subcategorias Capacidade de Fabricação e Medida de Desempenho.

Palavras-chave: Estratégia de Operações; Operações de Serviço; Operações no Setor Público.

\section{Strategies of service operations in the public sector: an examination of the Brazilian scientific production in the period 2004-2014}

This article aims to perform a systematic review of the Service Operations Management (SOM) field in the Public Sector from 2004 to 2014 in order to compile information on the characteristics and evolution of national publications related to the area. The survey results were divided into descriptive results and thematic results. The descriptive results aimed to answer four questions adapted from the model proposed by Machuca et al. (2007): (A) Which variations, if any, may have occurred in the number of publications in Service Operations in the Public Sector over the years? (B) What are the leading research topics? (C) Which methods are used in SOM research in the public sector? (D) What activity sectors are most commonly subjected to studying? The thematic results were based on the item classification according to their focal topic - content or process - as shown by Dangayach and Deshmukh (2001) and Boyer et al. (2005). Through descriptive results, it was verified a distribution of studies directed to the public sector following the general publications on SOM, planning being the main theme addressed, modeling confirmed as the most used method and the health sector acknowledged as the most studied among all. As for the thematic results the research showed a concentration of articles focusing on aspects of content with noticeable emphasis given to the subcategories Manufacturing Capacity and Performance Measurement.

Keywords: Operations Strategy; Service Operations; Operations in the Public Sector.

Topic: Produção e Operações

Reviewed anonymously in the process of blind peer.

Diego da Guia Santos

Universidade Federal de Alagoas, Brasil

http://lattes.cnpq.br/2442362471764000

diego.daguia@outlook.com

Flávio Henrique Reis Santos

Universidade Federal de Alagoas, Brasil

http://lattes.cnpq.br/8257177961608377

flavio@nti.ufal.br

Andrew Beheregarai Finger

Universidade do Vale do Rio dos Sinos, Brasil

http://lattes.cnpq.br/7035677257870103

finger.andrew@gmail.com

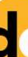

DOI: 10.6008/SPC2179-684X.2016.001.0011
Received: 09/08/2015

Approved: 18/01/2016
Referencing this:

SANTOS, D. G.; SANTOS, F. H. R.; FINGER, A. B.. Estratégias de operações de serviço no setor público: um exame a partir da produção científica brasileira no período de 2004-2014. Revista Brasileira de Administração Científica, v.7, n.1, p.160-175, 2016. DOI: http://doi.org/10.6008/SPC2179-684X.2016.001.0011 


\section{INTRODUÇÃO}

Os estudos sobre Estratégias de Operações de Serviços iniciam-se a partir dos trabalhos pioneiros sobre estratégia da Harvard Business School nos anos 50, chegando aos estudos mais recentes que procuram introduzir estratégias em gestão de operações relacionadas ao fornecimento de serviços, incluindo serviços oferecidos pelo poder público.

Partindo de modelos embrionários das décadas de 50 e 60, cujas formulações e implementações de práticas estratégicas orientavam-se para a ação, as pesquisas tornaram-se mais volumosas e consistentes e passaram a focalizar a aplicação da estratégia nas práticas organizacionais, visando a aperfeiçoar formas de produção e gestão e a alcançar níveis superiores de competitividade. Com a correlação entre estratégia e gestão de produção e operações foi possível compreender o potencial da área de gestão de operações dentro da organização, bem como analisar ambas as áreas a partir dos recursos e potencialidades que lhes são pertinentes, permitindo assim a elaboração de modelos de decisão e ação com o objetivo de incrementar as potencialidades de cada uma das áreas e de direcionar suas atuações para a criação e manutenção de vantagens competitivas.

Os estudos sobre Gestão de Operações em Serviços (GOS) intensificam-se a partir da década de 1970, mantendo uma forte ligação com os estudos preliminares sobre Gestão de Produção e Operações. Após incorporar a temática da qualidade disseminada na década de 80 , é nos anos 90 que o campo de serviços passa a integrar a lista de componentes fundamentais ao desenvolvimento de ações estratégicas para satisfazer as necessidades de clientes ou usuários, buscando os fidelizar: o foco no cliente e a capacitação de colaboradores organizacionais objetivavam o desenvolvimento de vantagens competitivas e a máxima satisfação do cliente. Os desenvolvimentos tecnológicos, as pesquisas mais aprofundadas e abrangentes, a personalização de serviços marcam a passagem da abordagem de Gestão de Operações em Serviços para o século XXI, implicando a introdução crescente de planejamento e desenvolvimento de estratégias para entregar aos clientes o melhor serviço com o menor custo, de forma a individualizar o cliente em suas necessidades e potencializar as entregas dos serviços criando um ambiente favorável de manutenção de altos níveis competitivos.

No Brasil as primeiras manifestações estruturadas da Gestão de Operações de Serviços ocorreram na década de 90, com a diminuição das barreiras alfandegárias, revisão das reservas de mercado e maior integração da economia brasileira com a economia globalizada.

$\mathrm{Na}$ oferta de serviços públicos, as crescentes demandas dos cidadãos para o atendimento de suas necessidades e a necessidade de racionalização de recursos públicos têm forçado governos através de seus órgãos de Administração Pública a agirem de forma cada vez mais eficiente e eficaz, buscando implementar ações afirmativas para oferecer serviços de qualidade e atingir resultados efetivos com impactos estruturais e infraestruturais relevantes. Assim, a formulação e implementação de ações estratégicas que dinamizem a Gestão de Operações de Serviços no Serviço Público podem permitir o desenvolvimento de ferramentas promissoras para o alcance dos objetivos governamentais. 
Visando a analisar o objeto de estudo proposto, em sequência a esta Introdução segue seção contendo referencial teórico que avalia a evolução da pesquisa sobre Estratégia de Operações, Gestão de Operações de Serviços e, finalmente, Estratégia de Operações de Serviços no setor público. A seção seguinte apresenta a abordagem metodológica empregada para coleta e avaliação dos artigos. A seção intitulada Resultados apresenta os resultados mais importantes verificados na pesquisa, bem como alguns pontos de limitação identificados no processo. A seção Considerações Finais apresenta um quadro sintético da realidade da pesquisa científica sobre o tema proposto, bem como sugestões que auxiliem na indicação de caminhos para futuros estudos sobre o tema.

\section{REVISÃO TEÓRICA}

\section{Estratégia de Operações}

As manifestações ocidentais mais consistentes de estudos sobre estratégia surgem nos anos 50 na Havard Business School, quando pesquisadores buscaram entender a razão do sucesso das empresas, o qual não parecia obedecer a regras claras do mercado (HAFSI e MARTINET, 2008). Para tal, os estudiosos se basearam na teoria rústica de Hipócrates que inspirou o método de casos (HAFSI e MARTINET, 2008). Com base em Hipócrates, amadureceu-se o entendimento de identificação de múltiplos aspectos que influenciavam em uma determinada situação. Estes aspectos seriam submetidos à discussão a fim de se esclarecer e compreender a problemática, objetivando-se a elaboração de teorias cuja finalidade seria a ação, definindo assim os rudimentos da estratégia corporativa (LEARNED et al. 1965).

Foi Andrews, nos anos 60, que conceituou e popularizou um modelo de avaliação de casos complexos denominado Conceito de Estratégia Corporativa de Harvard (ANDREWS, 1987), orientado para dois segmentos de análise: um segmento de formulação prática da estratégia e um segmento de implementação baseado na estrutura existente para a aplicação da estratégia. Paralelamente, Ansoff (1965) desenvolveu outra versão de um modelo mais fragmentado, porém mais concreto e preciso que o de Andrews, cujo foco eram os desafios estratégicos.

Foi com o trabalho seminal de Wickham Skinner publicado em 1969 na Harvard Business Review que a área de Estratégia de Operações começou a ser desenvolvida dentro dos estudos organizacionais. Nesta obra, Skinner defendia a existência de trade-offs operacionais determinantes das prioridades competitivas da produção.

Slack et al. (2008, p. 58) definem estratégia de operação como sendo "o modelo de decisões e ações que formatam a visão de longo prazo, os objetivos e as capacidades da operação e sua contribuição para a estratégia global". Este modelo envolve o desenvolvimento, posicionamento e alinhamento de políticas gerenciais e recursos necessários de forma que, além de contribuir, eles sejam consistentes com a estratégia organizacional global (ANDERSON, CLEVELAND e SCHROEDER, 1989; SKINNER, 1969; SWAMIDASS, 1986).

O direcionamento logicamente planejado das ações, alinhado às finalidades esperadas das operações, deve fomentar a criação de vantagens competitivas sustentáveis para a organização (MAIA et al., 
2005). Como a área de operações tem amplo alcance dentro do âmbito organizacional e considerando que a unidade de análise da estratégia de operações mudou da organização para a rede de organizações e a cadeia de suprimento (VOSS, 2005), ações funcionais de estratégia integrativas são determinantes para a criação e sustentação de valor competitivo (GARTNER e GARCIA, 2005). Tal alcance, segundo Lowson (2001), faz com que a estratégia de operações exerça influência: 1) nas decisões relacionadas ao foco de competências, potencialidades e processos; 2) nos recursos; 3 ) nas atividades táticas primordiais.

Com esta compreensão da abrangência peculiar à estratégia de operações, é possível indicar três funções básicas que deve exercer. A primeira seria alinhar e articular todos os processos e operações da organização com o objetivo de convergirem para o desenvolvimento e consolidação da estratégia organizacional global (SKINNER, 1969). Na literatura referente à estratégia de negócios, é reconhecido que uma estratégia de uma companhia pode não ser sempre completamente planejada, mas pode consistir de componentes planejados e emergentes (MINTZBERG, 1978). De fato, Mintzberg (1987) propôs que estratégias emergentes aliadas a planos proativos determinam a direção da estratégia global da organização.

Além disso, uma estratégia de operações deve ser criteriosamente elaborada visando identificar e definir os objetivos motivadores do desempenho das operações, bem como o conjunto decisório pertinente que promoverá o alcance destes objetivos pela organização através da execução operacional (HAYES, 1992; ST. JOHN e YOUNG 1992; VOSS e WINCH, 1996). Conforme asseveram Boyer et al. (2005), o conjunto decisório envolverá vários componentes da organização deliberando em consenso, visto que a área de operações requer entradas de uma grande proporção da organização.

Em terceiro lugar, o ponto mais importante e que é na verdade uma conciliação dos outros dois pontos: como utilizar os recursos de operações para que os requisitos do mercado - satisfação do cliente ou usuário seja satisfeita aliada à manutenção e ao aumento da competitividade organizacional - sejam realizados. $\mathrm{O}$ entrecorte conciliatório será originado da consonância entre os objetivos de desempenho da organização e as decisões adotadas com base na estratégia de operação. Quanto melhor for a associação de cada objetivo com uma estratégia operacional para sua execução, maior será a coerência do planejamento estratégico das operações (VOSS, 2005; ROTH e MILLER, 1992). Contingências nessa relação devem gerar a reavaliação estrutural do planejamento estratégico buscando-se a "reconciliação das ligações planejadas entre cada objetivo de desempenho e cada área de decisão" (SLACK et. al, 2008).

A estratégia de operações por si só não garante diferencial competitivo a todas as organizações que desenvolvem planejamento e gerenciamento estratégico. Organizações efetivas ou mais bem sucedidas são aquelas que conseguem atingir um bom nível de alinhamento entre a estratégia e a estrutura organizacional (MINTZBERG, 2006). Segundo Porter (2001), efetividade operacional e posicionamento estratégico são as formas de se alcançar a vantagem competitiva diferenciadora.

Decorridos mais de quarenta e cinco anos desde a proposta inicial de Skinner, as pesquisas organizacionais vêm, crescentemente, agregando conteúdos às discussões referentes à Estratégia de Operações, com a influência das cadeias de suprimento no ciclo estratégico e a contribuição de modelos 
como a Visão Baseada em Recursos, que fornece uma fundamentação teórica sólida na compreensão do papel da estratégia de operações na criação e manutenção da vantagem competitiva. (BHARADWAJ, 2000).

\section{Gestão de Operações de Serviço}

Nos últimos anos, o campo de serviços tem ganhado destaque escalar dentro da área de Gestão de Operações. Tal realidade teve início quando pesquisadores e profissionais perceberam a crescente participação de empresas não-manufatureiras no mercado e a carência de conhecimento e ferramentas para gerir tais empresas (JOHNSTON, 1999). Se inicialmente os trabalhos na área de serviços, inclusive os mais reconhecidos como medicina e odontologia, não tinham tanto prestígio, a partir do século 20 , principalmente na segunda metade, há uma mudança nesse cenário que fez com que o setor alcançasse o patamar de líder da economia de países como os Estados Unidos (HEINEKE e DAVIS, 2007).

Devido ao crescimento da temática de serviços na economia, pesquisadores desenvolveram um campo de estudo voltado para o setor, sendo possível já na década de 60 identificar a criação de modelos para otimização das atividades em alguns segmentos (HEINEKE e DAVIS, 2007). Nesse momento, a área que recebeu maior atenção foi a da saúde, como pode ser observado nos estudos de Long e Feldstein (1967), Shuman et al. (1971), Abernathy et al. (1971) e Abernathy e Hershey (1972). Long e Feldstein (1967) utilizam conceitos da teoria de filas para analisar o setor de obstetrícia de um hospital. Já Shuman et al. (1971) propõem um modelo que determina a combinação necessária entre mão de obra e tecnologia para prestar serviços de qualidade na área de saúde a um custo total mínimo. Abernathy et al. (1971) desenvolveram um modelo de agendamento para enfermeiros que estabelece equilíbrio entre o tamanho do setor de enfermagem e a demanda de pacientes enquanto Abernathy e Hershey (1972) analisaram a questão da localização de unidades de saúde.

Para entender o avanço das pesquisas em GOS ao longo das décadas, Heineke e Davis (2007) fizeram uma revisão dos estudos abordando os principais autores e foco dos trabalhos no decorrer dos anos.

Na primeira década (1970), apesar da busca pela especificidade do serviço, ainda existia muita ligação aos tradicionais temas abordados na produção fabril como processamento em lote, filas especializadas versus gargalos e planejamento de capacidade agregada. Dentre os principais trabalhos da época Heineke e Davis (2007) destacaram autores como Levitt e Sasser. Levitt (1972) traz algumas peculiaridades do setor de serviços e desmitifica alguns paradigmas relacionados à qualidade e responsabilidade dos profissionais envolvidos na área. Sasser (1976) discute duas estratégias básicas ("match supply" e "demand in service") disponíveis para a maioria das empresas de serviço. $\mathrm{O}$ autor continua debatendo algumas formas em que os gerentes de serviço podem alterar a demanda e influenciar a capacidade. Enquanto Sasser (1980b) traz o caso de sucesso da Burger King inserido neste cenário.

Na próxima década, ainda segundo Heineke e Davis (2007), as pesquisas e o ensino em serviços acompanharam o movimento da qualidade nos Estados Unidos. Nesse momento merece destaque um estudo de Parasuraman et al. (1985), que propõe um modelo conceitual de qualidade de serviço baseado nos resultados de um survey. 
Na terceira década (1990), o setor de serviço assume definitivamente o papel estratégico com foco na fidelização do cliente (HEINEKE e DAVIS, 2007). Schlesinger e Heskett (1991) citam que o pensamento industrial na área de serviço está obsoleto, destacando o surgimento de um modelo com foco no cliente e investimento nos funcionários como diferencial competitivo. Já Chase e Garvin (1989) identificaram várias abordagens de serviço que empresas de manufatura poderiam adotar para aumentar a sua competitividade.

A passagem para o século XXI marca o início da quarta década, com forte influência da tecnologia e serviços cada vez mais personalizados às necessidades dos clientes (HEINEKE e DAVIS, 2007).

Johnston (1999) também destaca quatro fases no processo de evolução das pesquisas em GOS. A primeira, de natureza descritiva e com foco em bens e serviços, é reconhecida pelo rompimento com pensamento fabril e conscientização da importância do serviço e contato com o cliente. A segunda possui uma vertente mais conceitual, sendo caracterizada pelo desenvolvimento de modelos para ajudar a compreender as características do serviço e gerenciamento de serviços. A terceira fase tem um caráter empírico, baseada na aplicação de modelos e desenvolvimentos de processos de serviço, aliados a conceitos como qualidade e tecnologia. Na quarta e última fase, de caráter prescritivo, o autor destaca o retorno às raízes conceituais para agregar mais profundidade à temática.

Como resultado da relevância econômica e acadêmica do campo de serviço, alguns pesquisadores desenvolveram revisões, a fim de analisar o avanço das temáticas específicas de GOS, destacando dentre eles os trabalhos de Machuca et al. (2007) e Mabert (1982). No entanto, em grande parte dos estudos a análise de serviços é restrita ao número total de publicações ao longo dos anos (BUFFA, 1980; AMOAKO-GYAMPAH e MEREDITH, 1989; PANNIRSELVAM et al., 1999).

No Brasil, as atenções para GOS ocorreram somente na década de 90 com a diminuição das barreiras alfandegárias e maior integração da economia brasileira com a economia mundial (CORREA et al., 2010). Como ocorrera no cenário internacional, alguns estudos nacionais buscaram destacar a evolução da área de operações fazendo referência ao avanço do campo de serviço (ARKADER, 2003; CORREA et al. 2010; PAIVA E BRITO, 2013).

\section{Estratégia de Operações de Serviço no Setor Público}

Tendo em vista a relevância econômica e acadêmica que a temática de GOS alcançou ao longo dos anos, a amplitude de suas aplicações se estendeu também ao setor público.

No ano de 2003, o campo de operações no serviço público foi pauta de uma edição especial de um periódico de alto impacto internacional (Journal of Operations Management), dada a importância que o tema vinha alcançando (VERMA et al. 2005).

No Brasil, o período marcado pelo desenvolvimento dos estudos em GOS coincide com o momento em que ocorre, segundo Bresser Perreira (1988), a reforma da Administração Pública brasileira, no qual o governo central passou a pautar suas ações orientado pelo paradigma do gerencialismo, com uma conotação mais empresarial em detrimento do paradigma burocrático anterior, fruto da aplicação da Teoria da 
Burocracia de Max Weber (WEBER, 1952), implementado no Brasil em substituição ao arcaico patrimonialismo administrativo.

A proposta da Administração Pública Gerencialista envolve uma mudança na estratégia de gerência (BRESSER PEREIRA, 1988) e busca encontrar respostas para os problemas da provisão de serviços públicos, e, em particular, dos serviços sociais, a partir de mudanças na organização e no funcionamento do aparelho burocrático do Estado (MORALES, 1998).

Bresser Pereira (1998) argumenta que as instituições públicas deveriam adotar o planejamento estratégico com vistas a otimizar os resultados de suas ações governamentais e atender às necessidades do ambiente de forma efetiva. Assim, o planejamento estratégico ocupa papel preponderante no diagnóstico, implementação e consecução das ações públicas, com o fim de atender às demandas sociais, aplicando práticas e ferramentas de gestão capazes de melhorar o desenvolvimento da estrutura e infraestrutura necessárias à operacionalização dos serviços públicos, pois, conforme defende Moore (1998), qualquer estratégia institucional do setor público deve produzir valor público, gerar resultados, ter legitimidade política suficiente para se sustentar e ser administrativa e operacionalmente viável.

Analisando especificamente a área de operações é possível identificar estudos que buscam analisar e melhorar a operacionalização dos serviços públicos em detrimento da qualidade, sejam eles no campo da saúde (CHIYOSHI, e MORABITO, 2010; SCARPIN et al., 2008), energia (PESSANHA et al. 2007; TOLEDO et al., 2008), educação (EBERLE et al. 2010; TOMASSINO et al. 2008), entre outros.

Neste contexto, pode-se verificar pelos artigos coletados para essa revisão sistemática que os pesquisadores estão sensíveis à necessidade de analisar possibilidades de associar estudos de Estratégia à área de GOS em Serviços Públicos como forma de disponibilizar o conhecimento organizacional da área de estratégia para os gestores públicos, para que os serviços oferecidos pela Administração Pública possam ser efetuados de forma mais eficiente, eficaz, menos onerosa, atendendo ao interesse público e ao bem comum. O esforço governamental para melhorar a qualidade dos gastos correntes com bens e serviços no setor público deve passar pela modernização da gestão de operações na oferta de serviços aos cidadãos.

\section{METODOLOGIA}

Santos e Finger (2015) realizam levantamento da produção nacional de GOS e identificaram uma predominância de trabalhos com foco público. Tais resultados motivaram o recorte específico para o setor público através do presente estudo, que tem como objetivo realizar uma revisão sistemática do campo de Gestão de Operações de Serviço no setor público com base nas publicações em revistas nacionais de alto impacto feitas no período de 2004 a 2014. Sampaio e Mancine (2007) afirmam que esse tipo de investigação disponibiliza um conjunto das evidências relacionadas a uma estratégia de intervenção particular, mediante a utilização de métodos explícitos e sistematizados de busca, apreciação crítica e síntese da informação desejada. Já Petticrew e Roberts (2006) destacam a importância de tais revisões para mapeamento de áreas nas quais verifica-se quantidade insuficiente estudos. 
Para garantir a relevância das publicações, foram selecionados periódicos classificados como A1 e A2 no cadastro Qualis/Capes na área de Administração. Como não havia revistas nacionais qualificadas como A1, o estudo se baseou nas seguintes revistas: Brazilian Administration Review (BAR), Brazilian Business Review (BBR), Contabilidade \& Finanças, Dados, Estudos Avançados, Gestão \& Produção, Opinião Pública, Organização \& Sociedade, Pesquisa Operacional, Revista de Administração Contemporânea (RAC), RAC Eletrônica, Revista de Administração de Empresas (RAE), RAE Eletrônica, RAUSP-e, Revista Brasileira de Economia, Revista de Administração e Revista de Administração Pública (RAP). Para os periódicos que tinham versão impressa e on-line foi priorizada a versão on-line.

A coleta dos artigos foi feita por meio de um levantamento nos volumes de cada periódico dentro do período estabelecido para análise. Logo após foi feita uma pesquisa na base Scielo de cada revista utilizando as keywords "operacoes" e "servico" com o conectivo "and" e posteriormente "opera\$" e "servi\$", também com o conectivo "and". No total forem levantados 49 artigos de GOS, sendo 21 voltados exclusivamente para o setor público.

Os resultados da pesquisa serão divididos em resultados descritivos e resultados temáticos. Os descritivos buscaram responder quatro questões adaptadas do modelo proposto por Machuca et al. (2007), com foco no setor público: (a) Que variações, se houver, podem ter ocorrido no número de publicações em Operações de Serviço no setor público no decorrer dos anos?; (b) Quais são os principais temas de pesquisa?; (c) Que métodos são usados em pesquisas de GOS no setor público?; (d) Quais os setores de atividade, se houver, são mais estudados?

Os resultados temáticos têm como ponto de partida a separação dos artigos segundo seu tópico focal - conteúdo ou processo - conforme apresentado por Dangayach e Deshmukh (2001) e Boyer et al. (2005). Boyer et al. (2005) definem as pesquisas com foco no conteúdo como sendo aquelas que referem-se ao estudo das capacidades e prioridades competitivas das operações, bem como opções e configurações estruturais (por exemplo, capacidade, tecnologia) e infraestruturais (por exemplo, força de trabalho). Enquanto escolhas estruturais referem-se às decisões reais, tangíveis, escolhas infraestruturais estão relacionadas às políticas e sistemas de gerenciamento dos componentes estruturais. Pesquisa com foco no processo refere-se ao estudo da tomada de decisão e desenvolvimento da estratégia, comunicação de decisões e implementação da estratégia (BOYER et al. 2005).

Já Dangayach e Deshmukh (2001) afirmam que a pesquisa com foco no conteúdo aborda questões relacionadas com prioridades competitivas, as quais incluem aspectos de custo, qualidade, confiabilidade e velocidade de entrega, flexibilidade e inovação, enquanto que processo é um padrão ou procedimento em que a estratégia de produção é desenvolvida e implementada.

A segunda análise dos resultados temáticos terá como base as subcategorias do tópico focal “conteúdo" apresentadas por Dangayach e Deshmukh (2001). Assim, os artigos levantados na presente pesquisa serão distribuídos nas seguintes subcategorias: Capacidade de Fabricação, Escolhas Estratégicas, Melhores Práticas, Comparação Transnacional, Mensuração de Desempenho e Levantamento Bibliográfico. 


\section{RESULTADOS}

Os temas Gestão de Operações e Gestão de Operações de Serviço foram pauta de várias revisões que buscaram descrever a evolução e características do campo (BUFFA, 1980; AMOAKO-GYAMPAH e MEREDITH, 1989; PANNIRSELVAM et al., 1999; ARKADER, 2003; CORREA et al., 2010; PAIVA E BRITO, 2013). Porém, pouca atenção foi dada a análises específicas do setor público. Essa variável está presente no trabalho de Mabert (1982), que identificou que $52,3 \%$ das publicações estavam voltadas para área pública e $47,7 \%$ para o setor privado, sugerindo um equilíbrio nos interesses entre os setores público e privado em temas de gestão de operações do período estudado.

O presente trabalho identificou 48 artigos publicados na área de GOS entre os anos de 2004 e 2014. Desses, $44 \%$ estavam focados no setor público, enquanto $35,4 \%$ no privado, $16,7 \%$ relacionavam público e privado, e 4,2\% não se aplicavam a tal categorização por se tratarem de trabalhos conceituais. Para entender a relação entre os estudos voltados para o campo público e o geral foi feita uma distribuição ao longo dos anos que pode ser observada no Gráfico 1.

Percebe-se que o número de artigos com foco público acompanhou a mesma tendência das publicações de GOS. Tal resultado é reflexo do predomínio dos trabalhos do campo público entre os autores nacionais, como já foi destacado anteriormente, o que reforça a importância do setor para a área de GOS.

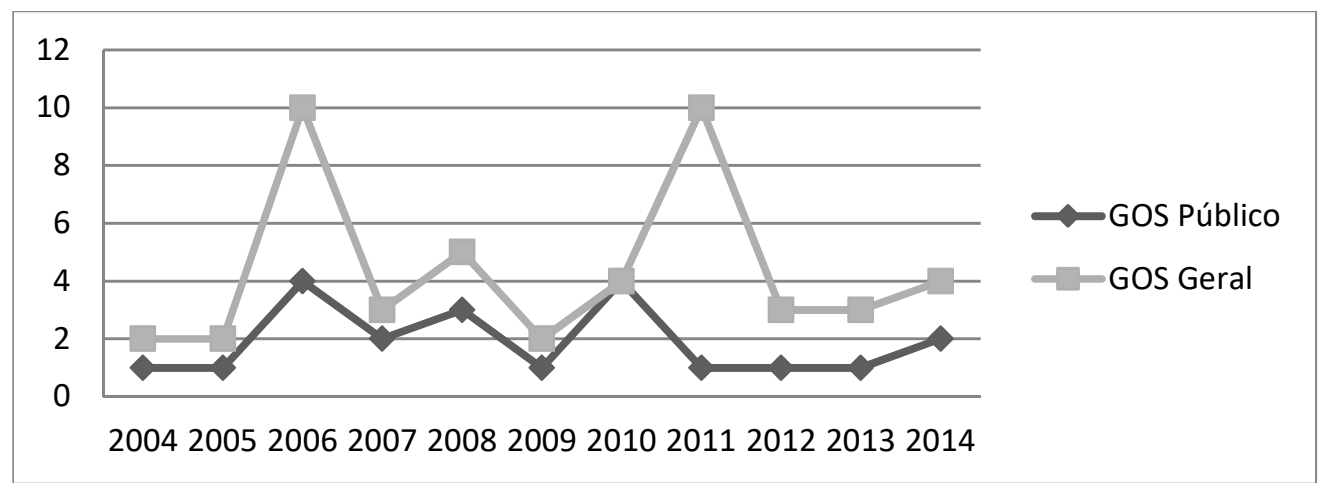

Gráfico 1: Relação entre publicação no setor público e publicação geral.

Outro recorte que pode ser feito é a distribuição anual por revista (Tabela 1). Nesse ponto, constatase um predomínio de periódicos que possuem linhas de pesquisa voltadas para operações, que têm seus trabalhos realizados por autores participantes de programas de pós-graduação de Administração, bem como programas da área de Engenharia de Produção (CORREA et al., 2010). Percebe-se também na Tabela 1, uma pequena oscilação no número de publicações ao longo dos anos, acompanhando proporcionalmente a produção em GOS, conforme verificado no Gráfico 1. Vale destacar que as revistas BAR, BBR, Dados, Estudos Avançados, Opinião Pública, RAC, RAC-e, RAE, RAUSP-e e Revista Brasileira de Economia não apresentaram publicações no período analisado.

Tabela 1: Distribuição anual por periódico.

\begin{tabular}{lcccccccccccc}
\hline \multicolumn{1}{c}{ Periódico } & $\mathbf{2 0 0 4}$ & $\mathbf{2 0 0 5}$ & $\mathbf{2 0 0 6}$ & $\mathbf{2 0 0 7}$ & $\mathbf{2 0 0 8}$ & $\mathbf{2 0 0 9}$ & $\mathbf{2 0 1 0}$ & $\mathbf{2 0 1 1}$ & $\mathbf{2 0 1 2}$ & $\mathbf{2 0 1 3}$ & $\mathbf{2 0 1 4}$ & Total \\
\hline Contabilidade \& Finanças & 0 & 0 & 0 & 1 & 0 & 0 & 0 & 0 & 0 & 0 & 0 & 1 \\
Gestão e Produção & 0 & 1 & 1 & 0 & 1 & 1 & 1 & 0 & 0 & 1 & 0 & 6 \\
O\&S & 0 & 0 & 0 & 0 & 0 & 0 & 0 & 0 & 1 & 0 & 0 & 1
\end{tabular}




\begin{tabular}{|c|c|c|c|c|c|c|c|c|c|c|c|c|}
\hline Pesquisa Operacional & 1 & 0 & 3 & 1 & 1 & 0 & 2 & 0 & 0 & 0 & 0 & 8 \\
\hline RAE-e & 0 & 0 & 0 & 0 & 1 & 0 & 1 & 0 & 0 & 0 & 0 & 2 \\
\hline RAP & 0 & 0 & 0 & 0 & 0 & 0 & 0 & 1 & 0 & 0 & 2 & 3 \\
\hline Total & 1 & 1 & 4 & 2 & 3 & 1 & 4 & 1 & 1 & 1 & 2 & 21 \\
\hline
\end{tabular}

O segundo ponto a ser observado nos resultados descritivos propostos pelo modelo de Machuca et al. (2007) está relacionado ao tema da pesquisa. A Tabela 2 demonstra uma superioridade de artigos voltados para planejamento e um número discreto de outros temas que foram destaque em outras décadas, como qualidade (9,5\%). Esse resultado vai ao encontro de outras revisões da área de GOS e operações. Arkader (2003), Paiva e Brito (2013) e Correia et al. (2010), ao analisarem a produção nacional em operações, observaram uma tendência para uma visão estratégica de operações e para a área de cadeia de suprimento, ao passo que a linha de qualidade se torna menos representativa.

Temas relacionados ao planejamento também são destaque nas publicações em GOS analisadas por Machuca et al. (2007), que verificaram o tema "desenho de serviço" como o mais abordado (9,3\%), seguido por "planejamento e controle em cadeias de suprimento" $(9,1 \%)$.

Tabela 2: Distribuição por temas.

\begin{tabular}{lccc}
\hline & Tema & Quantidade & Percentual \\
\hline Planejamento & & 10 & $47,6 \%$ \\
Avaliação de desempenho & 4 & $19,0 \%$ \\
Custos & & 2 & $9,5 \%$ \\
Qualidade & & 2 & $9,5 \%$ \\
Contratos & & 1 & $4,8 \%$ \\
Compras & & 1 & $4,8 \%$ \\
TOC & TOTAL & 1 & $4,8 \%$ \\
\hline
\end{tabular}

Quanto aos métodos utilizados, há um predomínio de estudos baseados em modelagem e simuladores (52\%). Já nos métodos empíricos destacam-se os trabalhos baseados em estudos de caso com 19\% (Tabela 3).

Tais resultados não corroboram com uma tendência destacada por autores como Machuca et al. (2007), que projetam uma diminuição dos estudos por modelagem e o aumento de métodos empíricos nas publicações em GOS. Esse destaque para métodos empíricos também é percebido por Arkader (2003) e Paiva e Brito (2013).

Tabela 3: Distribuição por método utilizado.

\begin{tabular}{|c|c|c|}
\hline Método & Quantidade & Percentual \\
\hline Modelagem e simuladores & 11 & $52 \%$ \\
\hline Estudo de Caso & 4 & $19 \%$ \\
\hline Survey & 2 & $10 \%$ \\
\hline Bibliográfico e Documental & 1 & $5 \%$ \\
\hline Documental & 1 & $5 \%$ \\
\hline Pesquisa-ação & 1 & $5 \%$ \\
\hline Survey e Bibliográfica & 1 & $5 \%$ \\
\hline Total & 21 & $100 \%$ \\
\hline
\end{tabular}

A última análise dos resultados descritivos está voltada aos setores que têm recebido maior atenção dos pesquisadores. A Tabela 4 apresenta um destaque para o campo da saúde (47\%). Tal setor mantém a 
situação de destaque em pesquisas anteriores de GOS como percebido em Machuca et al. (2007). Destaque inclusive já verificado nos levantamentos dos pesquisadores da primeira fase da gestão de operações de serviço (LONG e FELDSTEIN, 1967; SHUMAN et al. 1971; ABERNATHY e HERSHEY, 1972; ABERNATHY et al. 1971).

Tabela 4: Distribuição por setores.

\begin{tabular}{lccc}
\hline & Setor & Quantidade & \% \\
\hline Saúde & & 10 & $47,6 \%$ \\
Saneamento Básico & & 4 & $19,0 \%$ \\
Energia & & 2 & $9,5 \%$ \\
Educação & & 2 & $9,5 \%$ \\
Serviços de Massa/Lojas de serviço & 1 & $4,8 \%$ \\
Perícia Criminal & & 1 & $4,8 \%$ \\
Governo & Total & 1 & $4,8 \%$ \\
\hline & & $\mathbf{2 1}$ & $\mathbf{1 0 0 \%}$ \\
\hline
\end{tabular}

\section{DISCUSSÃO}

Para discorrer sobre tais resultados foi feito um recorte inicial específico dos artigos com base em seus respectivos tópicos focais (Tabela 5) segundo Dangayach e Deshmukh (2001) e Boyer et al. (2005). Percebe-se uma predominância de estudos com foco no conteúdo (71\%), o que corrobora com dados apresentados na pesquisa de Dangayach e Deshmukh (2001), que, ao realizar uma revisão sobre estratégia de operações no âmbito internacional, identificou um percentual de $91 \%$ para trabalhos direcionados ao conteúdo.

Tabela 5: Tópico Focal.

\begin{tabular}{lccc}
\hline & Foco & Quantidade & Percentual \\
\hline Conteúdo & & $\mathbf{1 5}$ & $\mathbf{7 1 \%}$ \\
Capacidade de fabricação & & 6 & $29 \%$ \\
Escolhas estratégicas & & 2 & $10 \%$ \\
Melhores práticas & & 1 & $5 \%$ \\
Comparação transacional & 0 & 0 & $29 \%$ \\
Mensuração de desempenho & & 6 & 0 \\
Levantamento bibliográfico & & 0 & $\mathbf{2 9 \%}$ \\
Processo & Total & $\mathbf{6}$ & $\mathbf{1 0 0 \%}$ \\
\hline
\end{tabular}

Com relação às subcategorias, o destaque fica por parte dos estudos direcionados à Capacidade de Fabricação e Mensuração de Desempenho, ambas com percentual de 29\%. Dangayach e Deshmukh (2001) identificaram a Capacidade de Fabricação como segunda subcategoria mais estudada, ficando atrás de Escolhas Estratégicas. Já Mensuração de Desempenho teve participação discreta.

Em seguida, os artigos levantados foram classificados segundo sua temática. Na Tabela 6 é possível identificar as principais contribuições percebidas nos estudos, além do tópico focal utilizado em cada trabalho. Percebe-se ainda na Tabela 6, um predomínio de artigos que fazem uso de modelos, como Hipercubo e PROMETHEE, para operacionalização do serviço proposto. 
Tabela 6: Classificação temática.

\begin{tabular}{|c|c|c|c|c|}
\hline Autor & Ano & Foco & Sub-foco & Principal contribuição \\
\hline $\begin{array}{l}\text { Takeda et } \\
\quad \text { al. }\end{array}$ & 004 & onteúdo & $\begin{array}{l}\text { Capacidade de } \\
\text { fabricação }\end{array}$ & $\begin{array}{l}\text { nalisou dos efeitos da descentralização de ambulâncias que operam no sistema } \\
\text { e atendimento médico-emergencial por meio do modelo Hipercubo. }\end{array}$ \\
\hline Sellito & 2005 & Conteúdo & $\begin{array}{l}\text { Mensuração } \\
\text { de } \\
\text { desempenho }\end{array}$ & $\begin{array}{l}\text { Discutiu o uso dos processos de pensamento da Teoria das Restrições como } \\
\text { alternativa para análise sistêmica em organizações. }\end{array}$ \\
\hline $\begin{array}{l}\text { lannoni e } \\
\text { Morabito }\end{array}$ & 2006 & Conteúdo & $\begin{array}{l}\text { Capacidade de } \\
\text { fabricação }\end{array}$ & $\begin{array}{l}\text { ombinou o modelo Hipercubo com um algoritmo genético para otimizar a } \\
\text { onfiguração e operação de sistemas de atendimento médico emergencial em } \\
\text { odovias. }\end{array}$ \\
\hline $\begin{array}{l}\text { lannoni e } \\
\text { Morabito }\end{array}$ & 2006 & Conteúdo & $\begin{array}{l}\text { Capacidade de } \\
\text { fabricação }\end{array}$ & $\begin{array}{l}\text { Aostrou como o modelo Hipercubo de filas pode ser modificado e aplicado para } \\
\text { nálise dos sistemas de atendimento médico emergencial em rodovias. }\end{array}$ \\
\hline $\begin{array}{l}\text { Marques e } \\
\text { Silva }\end{array}$ & 2006 & Conteúdo & $\begin{array}{l}\text { Mensuração } \\
\text { de } \\
\text { desempenho }\end{array}$ & $\begin{array}{l}\text { Discutiu a aplicação de uma técnica de cálculo da produtividade baseada no índice } \\
\text { de produtividade de Malmquist (IPM) aplicado aos serviços de água portugueses. }\end{array}$ \\
\hline $\begin{array}{l}\text { Morais et } \\
\text { al. }\end{array}$ & 2006 & Processo & Não se aplica & \\
\hline $\begin{array}{l}\text { Miranda et } \\
\quad \text { al. }\end{array}$ & 2007 & Conteúdo & $\begin{array}{l}\text { Melhores } \\
\text { práticas }\end{array}$ & valiou a utilização do Custeio $A B C$ nos Hospitais Universitários brasileiros. \\
\hline $\begin{array}{l}\text { Pessanha } \\
\text { et al. }\end{array}$ & 2007 & Conteúdo & $\begin{array}{l}\text { Mensuração } \\
\text { de } \\
\text { desempenho }\end{array}$ & $\begin{array}{l}\text { empenho } \\
\text { ssionárias }\end{array}$ \\
\hline $\begin{array}{l}\text { Toledo et } \\
\text { al. }\end{array}$ & 2008 & Conteúdo & $\begin{array}{l}\text { Capacidade de } \\
\text { fabricação }\end{array}$ & izar o custo de energia elétrica \\
\hline $\begin{array}{l}\text { Tomassino } \\
\text { et al. }\end{array}$ & 2008 & Conteúdo & $\begin{array}{l}\text { Mensuração } \\
\text { de } \\
\text { desempenho }\end{array}$ & $\begin{array}{l}\text { Investigou dois temas relacionados à análise das se } \\
\text { em expectativas e percepções ao longo do tempo } \\
\text { relação à futura qualidade do serviço - por meio de }\end{array}$ \\
\hline $\begin{array}{l}\text { Scarpin et } \\
\text { al. }\end{array}$ & 2008 & Processo & Não se aplica & $\begin{array}{l}\text { do do } \\
\text { ão do }\end{array}$ \\
\hline $\begin{array}{l}\text { Sakurada e } \\
\text { Miyake }\end{array}$ & 2009 & Processo & Não se aplica & $\begin{array}{l}\text { ou como } \\
\text { lo. }\end{array}$ \\
\hline $\begin{array}{l}\text { Rodrigues } \\
\text { et al. }\end{array}$ & 2010 & Conteúdo & $\begin{array}{l}\text { Capacidade de } \\
\text { fabricação }\end{array}$ & lo um processo de \\
\hline $\begin{array}{l}\text { Eberle et } \\
\text { al. }\end{array}$ & 2010 & Conteúdo & $\begin{array}{l}\text { Mensuração } \\
\text { de } \\
\text { desempenho }\end{array}$ & entes sobre os serviços de uma Instituição \\
\hline $\begin{array}{l}\text { Chiyoshi et } \\
\text { al. }\end{array}$ & 2010 & Processo & Não se aplica & 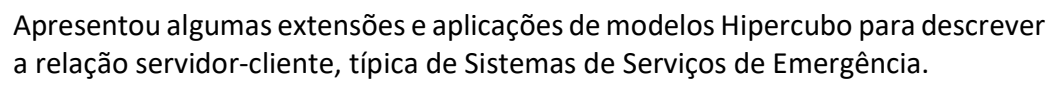 \\
\hline $\begin{array}{l}\text { Morais e } \\
\text { Almeida }\end{array}$ & 2010 & Processo & Não se aplica & $\begin{array}{l}\text { Apresentou um modelo dentro da abordagem multicritério fundamentada no } \\
\text { emprego do método PROMETHEE II, com o objetivo de contribuir para o } \\
\text { tratamento do problema da priorização de alternativas para redução de perdas e } \\
\text { desperdícios de água em sistemas de abastecimento. }\end{array}$ \\
\hline $\begin{array}{l}\text { Tridapalli } \\
\text { et al. }\end{array}$ & 2011 & Conteúdo & $\begin{array}{l}\text { Escolhas } \\
\text { estratégicas }\end{array}$ & $\begin{array}{l}\text { delo Conceitual e Operacional de gestão da cadeia de suprimento } \\
\text { co. }\end{array}$ \\
\hline $\begin{array}{l}\text { Luedy et } \\
\text { al. }\end{array}$ & 2012 & Conteúdo & $\begin{array}{l}\text { Escolhas } \\
\text { estratégicas }\end{array}$ & $\begin{array}{l}\text { Analisou em que medida o Contrato de Gestão vem alcançando resultados na } \\
\text { melhoria do desempenho de um Hospital Universitário }\end{array}$ \\
\hline $\begin{array}{l}\text { Souza et } \\
\text { al. }\end{array}$ & 2013 & Conteúdo & $\begin{array}{l}\text { Capacidade de } \\
\text { fabricação }\end{array}$ & $\begin{array}{l}\text { Verificou se o modelo hipercubo de filas espacialmente distribuídas é adequado } \\
\text { para analisar medidas de desempenho do SAMU/PR. }\end{array}$ \\
\hline $\begin{array}{l}\text { Bruns et } \\
\text { al. }\end{array}$ & 2014 & Conteúdo & $\begin{array}{l}\text { Mensuração } \\
\text { de } \\
\text { desempenho }\end{array}$ & $\begin{array}{l}\text { Verificou o desempenho de aspectos da assistência farmacêutica em municípios } \\
\text { da PB. }\end{array}$ \\
\hline $\begin{array}{l}\text { Heber e } \\
\text { Silva }\end{array}$ & 2014 & Processo & Não se aplica & $\begin{array}{l}\text { Avaliou o processo de institucionalização da Política Nacional de Resíduos Sólidos } \\
\text { (PNRS) a partir de um dos seus elementos estruturantes: a gestão compartilhada } \\
\text { dos resíduos sólidos. }\end{array}$ \\
\hline
\end{tabular}

Segundo Dangayach e Deshmukh (2001), estudos classificados em "Capacidade de Fabricação" incluem temas como as prioridades competitivas (custo, qualidade, entrega, flexibilidade); já "Escolhas Estratégicas" estão relacionadas a critérios estruturais e infraestruturais (recursos humanos, tecnologia, aspectos ambientais). Ainda segundo os autores, "Melhores Práticas" abrangem tecnologias avançadas de operações e melhores práticas de gestão, enquanto "Mensuração de Desempenho" envolve projetos de 
sistema, metodologias e avaliação. Por fim, Dangayach e Deshmukh (2001) trazem "Comparação Transacional" para falar de estudos multinacionais, e "Levantamento Bibliográfico" para artigos de revisão de literatura.

\section{CONSIDERAÇÕES FINAIS}

Nos últimos anos, o campo de serviços tem ganhado destaque escalar dentro da área Gestão de Operações. Tal realidade teve início quando pesquisadores e profissionais perceberam a crescente participação de empresas não-manufatureiras no mercado e a carência de conhecimento e ferramentas para gerir tais empresas (JOHNSTON, 1999).

Nesse contexto, particularizando para os serviços oferecidos pela Administração Pública em todas as suas esferas, percebe-se o esforço governamental em buscar a melhoria da qualidade dos serviços ofertados e maior racionalização dos gastos correntes necessários a oferta-los. Essa busca envolve a modernização da gestão de operações na oferta dos serviços aos cidadãos, e para isso, a introdução de conhecimentos de Estratégia de Operações de Serviços em Serviços Públicos tem-se mostrado promissora no alcance das metas governamentais.

Este artigo buscou verificar as características e evolução do tema Estratégias de Operações de Serviços em serviços públicos, como forma de avaliar a produção científica pertinente ao estabelecimento e implementação de modelos e ferramentas estratégicas em instituições públicas que oferecem serviços públicos aos cidadãos.

Por meio de resultados descritivos, verificou-se uma distribuição de trabalhos voltados para o setor público acompanhando as publicações gerais de GOS, tendo como principal tema abordado o planejamento, corroborando assim com estudos anteriores que destacam uma tendência para uma visão estratégica de operações e foco em planejamento, e desenho de serviço (ARKADER, 2003; PAIVA e BRITO, 2013; CORREIA et al., 2010; MACHUCA et al., 2007). Percebeu-se também um predomínio de estudos aplicados através de modelagem e simuladores, sendo esse um contraponto ao esperado por Machuca et al. (2007), quanto a expectativa de crescimento de estudos empíricos. No que se refere aos setores, a maior concentração de trabalhos esteve relacionada à área da saúde.

$\mathrm{Na}$ apresentação dos resultados temáticos ficou claro que houve uma concentração de artigos com foco em aspectos de conteúdo, com perceptível destaque para as subcategorias Capacidade de Fabricação e Medida de Desempenho. Considerando que a subcategoria Capacidade de Fabricação inclui estudos sobre qualidade e a categoria Medida de Desempenho aborda projetos de sistemas e metodologias de desenvolvimento e avaliação, a concentração verificada parece denotar que qualidade, desempenho e metodologias de replicação dos modelos e de avaliação dos serviços prestados são preocupações não só dos órgãos públicos e suas iniciativas, mas também dos pesquisadores, que estão alinhados com os objetivos da Administração Pública em prestar serviços públicos de qualidade.

Quanto às limitações do estudo, pode-se apontar o processo de categorização dos temas, visto que cada autor das referências que serviram de base para as comparações estabeleceu seus critérios e categorias. 
Além disso, alguns artigos coletados abordavam mais de um tema; esse estudo focou no tema central. Convém também ressaltar que algumas referências abordavam uma realidade internacional.

Como sugestão, futuras pesquisas em Gestão de Operações de Serviços na Administração Pública devem procurar diversificar as áreas de estudo, pois verificou-se com este artigo uma concentração em áreas da saúde, predominantemente. Áreas como Transporte Público, Educação Pública, Segurança Pública e definição de Políticas Públicas seriam imensamente beneficiadas com a introdução de conceitos e práticas de Estratégias de Gestão de Operações de Serviços específicas para a esfera pública e suas peculiaridades.

\section{REFERÊNCIAS}

ABERNATHY, W. J.; HERSHEY, J. C.. Spatial allocation model for regional services planning. Operations Research, v.20, n.3, p.620-642, 1972.

ABERNATHY, W. J.; BALOFF, N.; HERSHEY, J. C.. The nurse staffing problem: issues and prospects. Sloan Management Review, v.13, n.1, p.87-109, 1971.

ANDERSON, J. C.; CLEVELAND, G; SCHROEDER, R.. Operations strategy: A literature review. Journal of Operations Management, v.8, n.2, p.133-158, 1989.

AMOAKO-GYAMPAH, K.; MEREDITH, J. R.. The operations management research agenda: an update. Journal of Operations Management, v.8, n.3, p.250-262, 1989.

ANDREWS, K. R. The concept of corporate strategy. Homewood: Irwin, 1971.

ANSOFF, H. I.. Corporate strategy: an analytic approach to business policy for growth and expansion. New York: McGraw-Hill, 1965.

ARKADER, R.. A pesquisa científica em gerência de operações no Brasil. Revista de Administração de Empresas, v.43, n.1, p.70-80, 2003.

BHARADWAJ, A. S. .A resource-based perspective on information technology capability and firm performance: An empirical investigation. MIS Quarterly, v.24, n.1, p.169-196, 2000.

BOYER, K. K; SWINK, M; ROSENZWEIG, E. D.. Operations strategy research in the POMS Journal. Production and Operating Management, v.14, n.4, p.442-449, 2005.

BRESSER-PEREIRA, L. C.. Gestão do setor público: estratégia e estrutura para um novo Estado. In: BRESSER PEREIRA, L. C.; SPINK, P.. Reforma do Estado e administração pública gerencial. Rio de Janeiro: Editora da FGV, 1998.

BRUNS, S. F.; LUIZA, V. L.; OLIVEIRA, E. A.. Gestão da assistência farmacêutica em municípios do estado da Paraíba (PB): olhando a aplicação de recursos públicos. Revista de Administração Pública, v.48, n.3, p.745-765, 2014.

BUFFA, E. S.. Research in operations management. Journal of Operations Management, v.1, n. 1, p.1-8, 1980.
CHASE, R. B.; GARVIN, D. A.. The service factory. Harvard Business Review, v.67, n.4, p.61-69, 1989.

CHIYOSHI, F.; IANNONI, A. P.; MORABITO, R.. A tutorial on hypercube queueing models and some practical applications in emergency service systems. Pesquisa Operacional, v.31, n.2, p.271-299, 2010.

CORREA, H. L.; PAIVA, E. L; PRIMO, M. A.. A pesquisa em gestão de operações no Brasil: um breve relato de sua evolução. Revista de Administração de Empresa Eletrônica, v.9, n.2, p.1-9, 2010.

DANGAYACH, G. S.; DESHMUKH, S. G.. Manufacturing strategy. International Journal of Operations \& Production Management, v.21, n.7, p.884-932, 2001.

EBERLE, L.; MILAN, G. S.; LAZZARI, F.. Identificação das dimensões da qualidade em serviços: um estudo aplicado em uma instituição de ensino superior. RAE-eletrônica, v.9, n.2, 2010.

GARTNER, I. R.; GARCIA, F. G.. Criação de valor e estratégia de operações: um estudo do setor químico e petroquímico brasileiro. Gestão e Produção, v.12, n.3, p.459-468, 2005.

HAFSI, T.; MARTINET, A.-C.. Estratégia e Gestão Estratégica das Empresas: um Olhar Histórico e Crítico. Revista de Administração Contemporânea, Curitiba, v.12, n.4, p.11311158, 2008.

HAYES, R. H.. Production and operations management's new requisite variety. Production and Operations Management, v.1, n.3, p.249-253, 1992.

HEBER, F.; SILVA, E. M.. Institucionalização da Política Nacional de Resíduos Sólidos: dilemas e constrangimentos na Região Metropolitana de Aracaju (SE). Revista de Administração Pública, v.48, n.4, p.913-937, 2014.

HEINEKE, J; DAVIS, M. M.. The emergence of service operations management as an academic discipline. Journal of Operations Management, v.25, p.364-374, 2007.

IANNONI, A. P.; MORABITO, R.. Modelo hipercubo integrado a um algoritmo genético para análise de sistemas medicos emergenciais em rodovias. Gestão e Produção, v.13, n.1, p.93-104, 2006.

IANNONI, A. P.; MORABITO, R.. Modelo de fila hipercubo com múltiplo despacho e backupparcial para análise de 
sistemas de atendimento médico emergenciais em rodovias. Pesquisa Operacional, v.26, n.3, p.493-519, 2006.

JOHNSTON, R.. Service operations management: return to roots. International Journal of Operations \& Production Management, v.19, n.2, p.104-124, 1999.

LEARNED, E. P.; CHRISTENSEN, C. R.; ANDREWS, K. R.; GUTH, W. D.. Business policy: text and cases. Homewood: Irwin, 1965.

LEVITT, T.. Production-line approach to service. Harvard Business Review, v.50, n.5, p.41-52, 1972.

LONG, M. F.; FELDSTEIN, P. J.. Economics of hospital systems: peak loads and regional coordination. The American Economic Review, v.57, n.2, p.119-129, 1967.

LOWSON, R. H.. Retail operational strategies in complex supply chains. International Journal of Logistics Management, v.12, n.1, p.97-111, 2001.

LUEDY, A.; MENDES, V. L. P. S.; RIBEIRO JUNIOR, H.. Gestão Pública por Resultados: contrato de gestão como indutor de melhorias em um hospital universitário. Organização e Sociedade, v.19, n.63, p.641-659, 2012.

MABERT, V. A.. Service Operations Management: research and application. Journal of Operations Management, v.2, n.4, p.203-209, 1982.

MAIA, J.; CERRA, A.; ALVES FILHO, A.. Inter-relações entre Estratégia de Operações e Gestão de Cadeia de Suprimento: estudo de caso no segmento de motores para automóveis. Gestão e Produção, v.12, n.3, p.291-377, 2005.

MACHUCA, J. A. D; GONZÁLEZ-ZAMORA, M. M.; AGUILARESCOBAR, V. G.. Service Operations Management research. Journal of Operations Management, v.25, p.585-603, 2007.

MARQUES, R. C.; SILVA, D.. Análise da variação da produtividade dos serviços de água portugueses entre 1994 e 2001 usando a abordagem de Malmquist. Pesquisa Operacional, v.26, n.1, p.145-168, 2006.

MINTZBERG, H.. Patterns in strategy formation. Management Science, v.24, n.9, p.934-948, 1978.

MINTZBERG, H.. Crafting strategy. Harvard Business Review, p.66-75, 1987.

MINTZBERG, H.; LAMPEL, J.; QUINN, J.; GHOSHAL, S. O. Processo da Estratégia: conceitos, contextos e casos selecionados. Porto Alegre: Bookman, 2006.

MIRANDA, J. G.; CARVALHO, C. E.; MARTINS, F. V.; FARIA, A. F.. Custeio $A B C$ no Ambiente Hospitalar: um Estudo nos Hospitais Universitários e de Ensino Brasileiros. Contabilidade e Finanças, n.44, p.33-43, 2007.

MOORE, M. H.. Creating Public Value: strategic management in government. Harvard University Press. 1998.

MORAIS, D. C.; ALMEIDA, A. T.. Modelo de decisão em grupo para gerenciar perdas de água. Pesquisa Operacional, v.26, n.3, p.567-584, 2006.
MORAIS, D. C.; CAVALCANTE, C. A. V.; ALMEIDA, A. T.. Priorização de áreas de controle de perdas em redes de distribuição de água. Pesquisa Operacional, v.306, n.1, p.1532, 2010.

MORALES, C. A.. Nem privado nem estatal: em busca de uma nova estratégia para a provisão de serviços públicos. Revista do Serviço Público, v.49, n.4, 1998.

PAIVA, E. L; BRITO, L. A. L.. Produção científica brasileira em gestão de operações no período 2000-2010. Revista de Administração de Empresas, v.53, n.1, 2013.

PANNIRSELVAM, G.. Operations management research: an update for the 1990s. Journal of Operations Management, v.18, p.95-112, 1999.

PARASURAMAN, A.; ZEITHAML, V. A.; BERRY, L. L.. A conceptual model of service quality and its implications for future research. Journal of Marketing, v.49, n.4, p.41-50, 1985.

PESSANHA, J. F. M.; SOUZA, R. C.; LAURENCEL, L. C.. Um modelo de análise envoltória de dados para o estabelecimento de metas de continuidade do fornecimento de energia elétrica. Pesquisa Operacional, v.27, n.1, p.51-83, 2007.

PETTICREW, M.; ROBERTS, H.. Systematic reviews in the social sciences: a practical guide: Blackwell Publishing, 2006.

PORTER, Michael E.. Strategy and the Internet. Harvard Business Review, 2001.

RODRIGUES, C. V.; SILVA, M. T.; TRUZZI, O. M. S.. Perícia criminal: uma abordagem de serviços. Gestão e Produção, v.17, n.4, p.843-857, 2010.

ROTH, A.; MILLER, J.. Success factors in manufacturing. Business Horizons, p.73-81, 1992.

SAKURADA, N.; MIYAKE, D. I.. Aplicação de simuladores de eventos discretos no processo de modelagem de sistemas de operações de serviços. Gestão e Produção, v.16, n.1, p.2543, 2009.

SAMPAIO, R. F.; MANCINI, M. C.. Estudos de revisão sistemática: um guia para síntese criteriosa da evidência científica. Revista Brasileira de Fisioterapia, v.11, n.1, p.8389, 2007.

SANTOS, D. G.; FINGER, A. B.. Gestão de Operações de Serviço: Uma Revisão Sistemática sobre a Produção Nacional. In: ENCONTRO DA ANPAD, 39. Anais. Belo Horizonte, 2015.

SASSER JR., W. E.. Matching supply and demand in service industries. Harvard Business Review, v.54, n.6, p.132-138, 1976.

SASSER JR., W. E., RIKERT, D. C.. Burger King Corp. Harvard Business School Press, 1980b.

SCARPIN, C. T.; STEINER, M. T. A.; DIAS, G. J. C.; STEINER NETO; P. J.. Otimização no serviço de saúde no estado do Paraná: fluxo de pacientes e novas configurações hierárquicas. Gestão e Produção, v.15, n.2, p.275-290, 2008. 
SCHLESINGER, L. A.; HESKETT, J. L.. The service-driven service company. Harvard Business Review, 1991.

SELLITTO, M. A.. Processos de Pensamento da TOC como Alternativa Sistêmica de Análise Organizacional: Uma Aplicação em Saúde Pública. Gestão e Produção, v.12, n.1, p.81-96, 2005.

SHUMAN, L. J., YOUNG, J. P., NADDOR, E.. Manpower mix for health services: a prescriptive regional planning model. Health Services Research, v.6, n.2, p.103-119, 1971.

SKINNER, W.. Manufacturing: Missing link in corporate strategy. Harvard Business Review, v.47, n.3, p.136-145, 1969.

SLACK, N.; CHAMBERS, S.; JOHNSTONS, R.; BETTS, A.. Gerenciamento de operações e de processos: princípios e práticas de impacto estratégico. Porto Alegre: Bookman, 2008.

SOUZA, R. M.; MORABITO, R.; CHIYOSHI, F. Y.; IANNONI, A. P.. Análise da configuração de SAMU utilizando múltiplas alternativas de localização de ambulâncias. Gestão e Produção, v.20, n.2, p.287-302, 2013.

ST. JOHN, C. H.; YOUNG, S. T.. An exploratory study of patterns of priorities and trade-offs among operations managers. Production and Operations Management, v.1, n.2, p.133-150, 1992

SWAMIDASS, P. M.. Manufacturing strategy: Its assessment and practice. Journal of Operations Management, v.6, n.4, p.471-484, 1986.
TAKEDA, R. A.; WIDMER, J. A.; MORABITO, R.. Aplicação do modelo hipercubo de filas para avaliar a descentralização de ambulâncias em um sistema urbano de atendimento médico de urgência. Pesquisa Operacional, v.24, n.1, p.39-71, 2004.

TOLEDO, F. M. B.; SANTOS, M. O.; ARENALES, M. N.; SELEGHIM JÚNIOR, M.. Logística de distribuição de água em redes urbanas - racionalização energética. Pesquisa Opereacional, v.28, n.1, p.75-91, 2008.

TOMASSINI, R.; AQUINO, R.; CARVALHO; F. A.. Expectativas e percepções em sequências de serviço. RAE-eletrônica, v.7; n.2, 2008

TRIDAPALLI, J. P.; FERNANDES, E.; MACHADO, W. V.. Gestão da cadeia de suprimento do setor público: uma alternativa para controle de gastos correntes no Brasil. Revista de Administração Pública, v.45, n.2, p.401-433, 2011.

VERMA, R.; MCLAUGHLIN, C.; JOHNSTON, R.; YOUNGDAHL, W.. Operations management in not-for-profit, public and government services Charting a new research frontier. Journal of Operations Management, v.23, p.117-123, 2005.

VOSS, C. A.. Paradigms of manufacturing strategy re-visited International Journal of Operations \& Production Management, v.25, n.12, p.1223-1227, 2005.

VOSS, C. A.; WINCH, G. W.. Including engineering in operations strategy. Production and Operations Management, v.5, n.1, p.78-90, 1996.

WEBER, M.. The Essentials of Bureaucratic Organizations: an ideal-type construction. In: MERTON, R.. Reader in Bureaucracy: Glencoe III. The Free Press, p.18-27, 1952. 